\title{
Optional Inventory Ordering Policies of Multiple Non-Instantaneous Deteriorating Items Under Trade Credit and Price Discount
}

\author{
Kunxiang Dong ${ }^{1 *}$ \\ 1. School of Management Science and Engineering, \\ Shandong University of Finance and Economics,
} Jinan, China

\author{
Zongxiao $\mathrm{Xie}^{2}$ \\ 2. China Financial Certification Authority, \\ Beijing, China
}

\author{
Jie Zhen ${ }^{3}$ \\ 3. School of Business Planning, \\ Chongqing Technology and Business University, Chongqing, China \\ *Corresponding author: Kunxiang Dong
}

\begin{abstract}
When ordering multiple items, retailers tend to be used the collaborative procurement, so conducive to the formation of scale in quantities utility. Therefore, this paper established the inventory model of the retailer' in a multiple non-instantaneous deterioration items supply chain, with two kinds of incentives-credits and price discounts. Then we use the theorems to proof the existence of the optimal solution of inventory, and compare the profits under different scenarios. Finally, we use numerical examples to validate the model, we found that the optimal profits of retailer under trade credit is better than that of price discount in the multiple non-instantaneous deterioration items supply chain.
\end{abstract}

Keywords: Multiple non-instantaneous deteriorating items, trade credit, price discount, supply chain

\section{INTRODUCTION}

No-matter how big the company, they all face up two tough problems: the shortage of money and the inventory ordering policies of multiple non-instantaneous deterioration items with uncertainty demand. Especially, the extension of the concept of multiple non-instantaneous deterioration items is involving most of our daily products, with the development of society and technology. One of the most important branches of operation is the inventory model of multiple noninstantaneous deterioration items, and has been systematically summarized. But, in practice, retailers always order multiple non-instantaneous deterioration items, and the single item inventory model is not so precisely for the multiple non-instantaneous deterioration items. Therefore, to tackle the inventory ordering policies of multiple non-instantaneous deterioration items is aroused highly attention in the field of scholars. Bose et al. (1995) built up the EOQ model for deterioration goods with liner demand, allowing shortage and backlogging, and the influence of time value, firstly. And they acquire the inventory policies in difference scenarios. Moon et al. (2005) described a special kind of deterioration items, which the price goes up with time, for example, wine, poultry. And they also build up the inventory model with considering time value; Yang and Chang. (2013) formulated the optimal replenishment model by considering trade credit, time value, two warehouse and allowing shortage and backlogging; Tsao (2010) considered multi-echelon multi-item channels subject to supplier's credit period and retailer's promotional effort. They analyse two trade allowances, the promotion costs sharing and the cash discount, finding that coordination mechanism is better; Wen and $\mathrm{Da}$ (2006) discuss single ordering, partly ordering and coordination ordering from the perspective of retailer's, and they got the optimal ordering policies; Wang and Jiang (2007) compare four different circumstance of the combination of decentralized decision and cooperative decision, finding that the cooperative circumstance is better; Mo (2011) considered a model with demand depend on inventory and resisted space. They got the result by line search algorithm; Zhang et al. (2012) provide a completed literature review of the joint inventory policies of multi-deterioration items, and find out the inventory model of multiple deterioration items will be hot in the future.

Above all, they just consider the goods is deteriorating, when the goods arrive at the store, but, in practice, some kinds of goods have a certain life long time. So the existing models is not so precisely to tackle the inventory issues of noninstantaneous deterioration items. Wu (2006) provide the concept of non-instantaneous deterioration items, and build the related models to solve it; Chang et al. (2010) formulated the EOQ model of non-instantaneous deterioration items with considering constant demand, allowing shortage and backlogging, and the backlogging depends on the time of next replenishment time; Geetha et al. (2010) extent the model of Zhang et al.(2012), with considering demand depends on time and price, the room of retailer's shelves house and the maximize of the warehouse; Maihami et al. (2013a) considered the trade credit and partly backlogging, then Maihami et al. (2013b) extent their model with demand depend 
on time and price; Nita et al. (2013) assume that the demand function is rely on the advertisement and price. What's more, the model is considering a general holding cost function and a general deteriorating rate; Dye et al. (2013) established a maximize the profit model with considering constant demand, the effect of preserve technology, a generalized deterioration rate, and inventory and backlogging depends on time. They got the different factors' influence; Li and Wang (2011) built up the model of minimize the profit of retailer's, and analyse the influence of the time of beginning deterioration, deterioration rate and trade period.

Above all, deterioration items inventory research can be divided into two categories: deteriorating immediately and non instantaneous deterioration. Even there are some literatures have been studied the multi-deterioration items, but the research about non-instantaneous deterioration items with trade credit is rarely. We build up the inventory ordering model under price discount and trade credit to maximize the retailer's profit, and compare the two strategies, get that the profit under trade credit is better than that of under price discount in multiple non-instantaneous deterioration items supply chain.

\section{PROBLEM DESCRIPTION, ASSUMPTIONS AND NOTATIONS}

\subsection{Problem description}

In this paper, we consider the retailer how to make inventory policies in a multiple non-instantaneous deterioration items supply chain. In order opening, the retailer will order all the non-instantaneous deterioration items together, and at the end of ordering period, all products are already completely sold. Items have no effect to others. Meanwhile, suppliers will provide the trade credit or price discount to retailer, to stimulate the order quantity. Modelling the problem based on the process shown in Fig. 1:

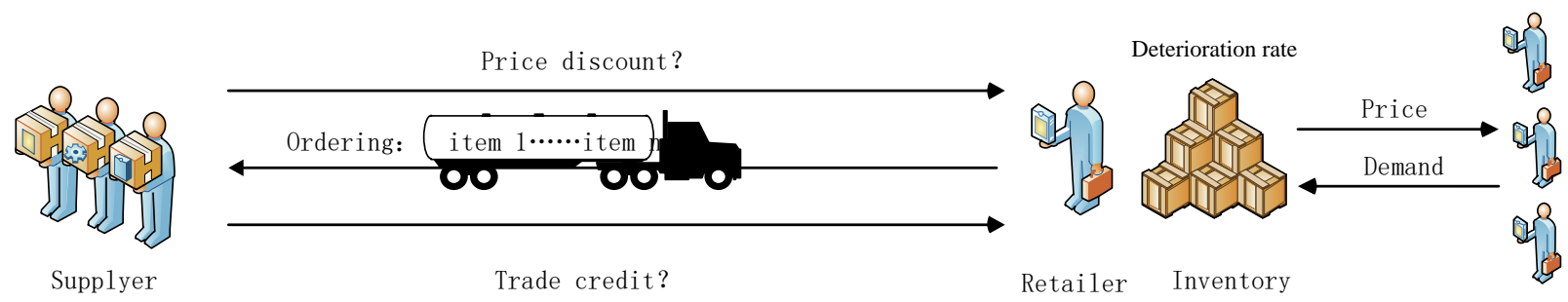

Fig. 1 The structure of modelling

\subsection{Assumptions}

(1)Replenishment rate is infant, lead time is zero, and no shortage are allowed.

(2)The demand of item $i$ depends on time and price, $D_{i}\left(p_{i}, t\right)=\left(a_{i}-b_{i} p_{i}\right) \mathrm{e}^{\lambda_{i} t}\left(a_{i}>0, b_{i}>0\right)$, in this paper we assume $\lambda_{i}<0$.

(3)Deterioration rate $\theta_{i}$ depends on inventory, and the beginning time of deterioration is smaller than ordering period $\max \left(t_{d i}\right)<T$.

(4) Bad items can't be repaired or replaced in the order cycle.

(5) Capital rate of inventory opportunity cost is greater than the current interest rates, $I_{p}>I_{e}$.

\subsection{Notations}

$S:$ the ordering cost per cycle, has nothing to do with the kinds of items per order.

$S_{i}$ : the additional ordering cost of item $i$, the particularity of the item, different item in the process of transportation, loading and unloading and additional costs.

$T$ : ordering period.

$c_{i}$ : the purchase cost of per unit item of $i$.

$h_{i}$ : the holding cost of per unit item of $i$.

$p_{i}:$ the price of item $i, p_{i}>c_{i}$.

$\theta_{i}$ : the deterioration rate of item $i$.

$t_{d i}$ : the beginning time of deterioration of item $i$.

$Q_{i}$ :the order quantity of item $i$.

$I_{l i}(t)$ : the inventory level of product $i$ during interval 1 at time $t$.

$I_{2 i}(t)$ : the inventory level of product $i$ during interval 2 at time $t$.

$I_{e}:$ the current interest rates.

$I_{p}$ : capital rate of inventory opportunity cost.

$r:$ discount rate. 
$T P_{j}$ : the retailer's total profit of circumstance $j, j=1,2,3$.

\section{Model formulation}

2.1 The model under price discount

First of all, the supplier provide price discount to retailer, retailers make his inventory policies by maximizing the profit. When , inventory system under the price discount is shown in fig.2:

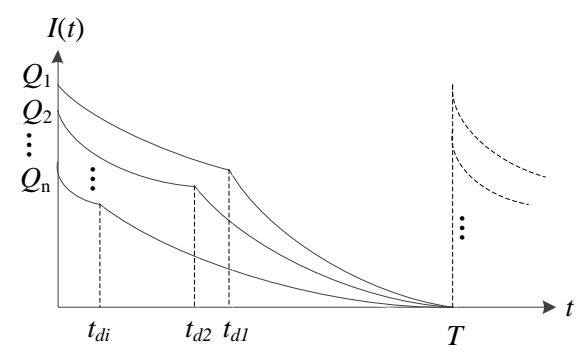

Fig. 2 Retailer's inventory levels under the price discount

The inventory goes like this: during the time interval $\left[0, t_{\mathrm{di}}\right]$, the inventory level is decreasing only owing to demand. The inventory level is dropping to zero due to demand and deterioration during the time interval [tdi, T].

Using the differential equation representing the inventory status is given by:

$$
\frac{d I_{I i}(t)}{d t}= \begin{cases}-\left(a_{i}-b_{i} p_{i}\right) \mathrm{e}^{\lambda_{i} t} & 0 \leq t \leq t_{d i} \\ -\left(a_{\mathrm{i}}-b_{\mathrm{i}} p_{i}\right) \mathrm{e}^{\lambda_{i} t}-\theta_{i} I_{2 i}(t) & t_{d i} \leq t \leq T\end{cases}
$$

According to the boundary conditions, yields:

$$
I_{i}(t)= \begin{cases}I_{l i}(t)=\frac{\left(a_{i}-b_{i} p_{i}\right)}{\lambda_{i}}\left[1-e^{\lambda_{i} t}\right]+Q_{i} & 0 \leq t \leq t_{d i} \\ I_{2 i}(t)=\frac{\left(a_{i}-b_{i} \mathrm{p}_{i}\right) e^{-\theta_{i} t}}{\lambda_{i}+\theta_{i}}\left[e^{\left(\lambda_{i}+\theta_{i}\right) T}-e^{\left(\lambda_{i}+\theta_{i}\right) t}\right] & t_{d i} \leq t \leq T\end{cases}
$$

Let $I_{1 i}\left(t_{d i}\right)=I_{2 i}\left(t_{d i}\right)$, we get:

$$
\begin{aligned}
Q_{i} & =\frac{\left(a_{i}-b_{i} p_{i}\right) e^{-\theta_{i} t_{d i}}}{\lambda_{i}+\theta_{i}}\left[e^{\left(\lambda_{i}+\theta_{i}\right) T}-e^{\left(\lambda_{i}+\theta_{i}\right) t_{d i}}\right]-\frac{\left(a_{i}-b_{i} p_{i}\right)}{\lambda_{i}}\left[1-e^{\lambda_{i} t_{d i}}\right] \\
& =\sum_{i=1}^{n}\left(a_{i}-b_{i} p_{i}\right)\left(T-\theta_{i} t_{d i} T+\theta_{i} t_{d i}^{2}\right)
\end{aligned}
$$

We have the retailer's total profit function:

$$
\begin{aligned}
& T P^{1}(p, T, R)=\sum_{i=1}^{n}[ p_{i}\left(a_{i}-b_{\mathrm{i}} p_{i}\right)-c_{i}(1-R)\left(a_{i}-b_{i} p_{i}\right)\left(1-\theta_{i} t_{d i}\right)-\frac{h_{i}\left(a_{i}-b_{i} \mathrm{p}_{i}\right)}{T} \frac{\left(1-2\left(\lambda_{i}+\theta_{i}\right) T\right) t_{d i}}{\lambda_{i}+\theta_{i}}- \\
&\left.c_{i} \theta_{i}(1-R)\left(\mathrm{a}_{i}-b_{i} p_{i}\right)\left(T-t_{d i}\right)-\frac{c_{i} I_{\mathrm{p}}(1-\mathrm{R})\left(a_{i}-b_{i} p_{i}\right)}{T} \frac{\left(1-2\left(\lambda_{i}+\theta_{i}\right) T\right) t_{d i}}{\lambda_{i}+\theta_{i}}\right]-\frac{1}{T}\left(S+\sum_{i=1}^{n} s_{i}\right)
\end{aligned}
$$

In the order of Eq.(4) : sales revenue of item $i$, purchase cost of item $i$, holding cost of item $i$, deterioration cost of item $i$, inventory opportunity cost of item $i$, ordering cost.

2.2 The model under trade credit

In the multiple non-instantaneous deterioration item inventory system, we build up the inventory model under three scenarios: (1) $M<t_{d i}<T$; (2) $t_{d i}<M<T$; and (3) $t_{d i}<T<M$, with the constrain of $T>t_{d i}$. The inventory system under the trade credit is shown in fig. 3 .

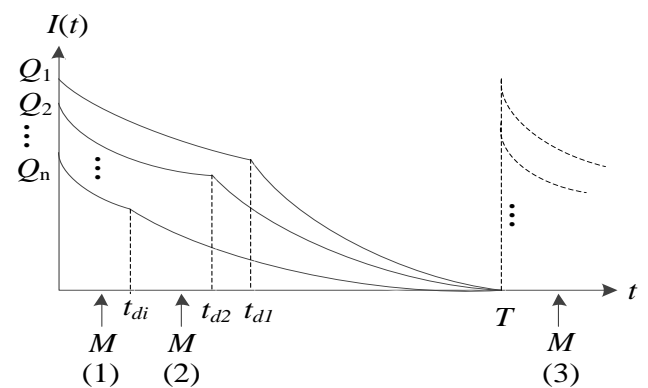

Fig. 3 Retailer's inventory levels under the trade credit 
According to Fig.3, we can get the retailer's total profit function, when $M<T$.

$$
\begin{aligned}
T P^{2}(p, T, R)= & \sum_{i=1}^{n}\left\{p_{i}\left(a_{i}-b_{\mathrm{i}} p_{i}\right)+\frac{p_{i} I_{e}\left(a_{i}-b_{i} p_{i}\right) M}{T}-c_{i}\left(a_{i}-b_{i} p_{i}\right)\left(1-\theta_{i} t_{d i}\right)(1-r M)-\right. \\
& \frac{h_{i}\left(a_{i}-b_{i} p_{i}\right)}{T} \frac{\left(1-2\left(\lambda_{i}+\theta_{i}\right) T\right) t_{d i}}{\lambda_{i}+\theta_{i}}-\frac{c_{i} \theta_{i}\left(a_{i}-b_{i} p_{i}\right)}{T\left(\lambda_{i}+\theta_{i}\right)}\left(t_{d i}-T\right)\left(2+\left(\lambda_{i}+\theta_{i}\right) T\right)- \\
& Y_{\mathrm{i}} \frac{c_{i} I_{\mathrm{p}}\left(a_{i}-b_{i} p_{i}\right)}{T}\left[\left(t_{d i}-M\right)\left(T-\theta_{i} t_{d i} T+\theta_{i} t_{d i}^{2}\right)+T\right]- \\
& \left.\left(1-Y_{\mathrm{i}}\right) \frac{c_{i} I \mathrm{p}\left(a_{i}-b_{i} p_{i}\right)(T-M)\left(1-\theta_{i}-r+\left(\lambda_{i}+\theta_{i}\right) T\right)}{T\left(\lambda_{i}+\theta_{i}\right)\left(\theta_{i}+r\right)}\right\}-\frac{1}{T}\left(S+\sum_{i=1}^{n} s_{i}\right)
\end{aligned}
$$

And $Y_{i}=\left\{\begin{array}{ll}1 & M<t_{d i}<T, \text { for item } i \\ 0 & t_{d i}<M<T, \text { for item } i\end{array}\right.$.

In the order of Eq.(5) : sales revenue of item $i$, interest revenue of item $i$, purchase cost of item $i$, holding cost of item $i$, deterioration cost of item $i$. when $M<t_{d i}<T$, the inventory opportunity cost of item I; when $t_{d i}<M<T$, the inventory opportunity cost of item $i$, and the ordering cost.

When $t_{d i}<T<M$, the retailer's profit function is:

$$
\begin{aligned}
T P^{3}(p, T, R)=\sum_{i=1}^{n} & {\left[p_{i}\left(a_{i}-b_{\mathrm{i}} p_{i}\right)+p_{i} I_{e}\left(a_{i}-b_{i} p_{i}\right)(1+T-M)-c_{i}\left(a_{i}-b_{i} p_{i}\right)\left(1-\theta_{i} t_{d i}\right)(1-r M)-\right.} \\
& \left.\frac{h_{i}\left(a_{i}-b_{i} p_{i}\right)}{T} \frac{\left(1-2\left(\lambda_{i}+\theta_{i}\right) T\right) t_{d i}}{\lambda_{i}+\theta_{i}}-\frac{c_{i} \theta_{i}\left(a_{i}-b_{i} p_{i}\right)}{T\left(\lambda_{i}+\theta_{i}\right)}\left(t_{d i}-T\right)\left(2+\left(\lambda_{i}+\theta_{i}\right) T\right)\right]- \\
& \frac{1}{T}\left(S+\sum_{i=1}^{n} s_{i}\right)
\end{aligned}
$$

In the order of Eq.(6) : sales revenue of item $i$, interest revenue of item $i$, purchase cost of item $i$, holding cost of item $i$, deterioration cost of item $i$, and the ordering cost.

Above all, the function (3)-(6) can be described as:

$$
T P=\left\{\begin{array}{lc}
T P^{1}(p, T, R) & \text { price discount } R \\
T P^{2}(p, T, M) & t_{d i}<T \& M<T \\
T P^{3}(p, T, M) & t_{d i}<T<M
\end{array}\right.
$$

3. Theoretical results

3.1 Results with price discount

We discuss the optimal ordering quantity and ordering period by maximizing the profit of retailer. As $p_{i}$ has the same identity and no effect to each other. We use $p$ to alternative $p_{i}$ for simple. First, we discuss the $(p, T)$ not only exist and the only, for any $R$. Then, we discuss the $R$ not only exist and the only, for any $p$ and $T$.

Taking the partial derivative of $T P^{1}$ with respective to $p$ and $T$, we have:

$$
\begin{aligned}
& \frac{\partial T P^{1}(p, T, R)}{\partial p}=\sum_{i=1}^{n}\left[a_{i}-2 b_{i} p_{i}+b_{i} c_{i}(1-R)\left(1-\theta_{i} t_{d i}\right)+\frac{b_{i} t_{d i}\left(1-2\left(\lambda_{i}+\theta_{i}\right) \mathrm{T}\right)}{T\left(\lambda_{i}+\theta_{i}\right)}\left(h_{i}+c_{i} I_{\mathrm{p}}(1-\mathrm{R})\right)+\right. \\
& \left.\quad b_{i} c_{i} \theta_{i}(1-R)\left(T-t_{d i}\right)\right] \\
& \frac{\partial T P^{1}(p, T, R)}{\partial T}=\frac{1}{T^{2}}\left(S+\sum_{i=1}^{n} s_{i}\right)+\sum_{i=1}^{n}\left[\frac{\left(a_{i}-b_{i} p_{i}\right) t_{d i}}{T^{2}\left(\lambda_{i}+\theta_{i}\right)}\left(h_{i}+c_{i} I_{\mathrm{p}}(1-R)\right)-c_{i} \theta_{i}(1-R)\left(a_{i}-b_{i} p_{i}\right)\right]
\end{aligned}
$$

Taking the second partial derivative of $T P^{1}$ with respective to $p$ and $T$, and we have the Hessian matrix:

$$
\begin{aligned}
\Delta\left(H^{1}\right) & =\left(\frac{\partial^{2} T P^{1}}{\partial p \partial T}\right)^{2}-\frac{\partial^{2} T P^{1}}{\partial p^{2}} \times \frac{\partial^{2} T P^{1}}{\partial T^{2}} \\
& =\left[\sum_{i=1}^{n}\left[\frac{-b_{i} t_{d i}}{T^{2}}\left(h_{i}+c_{i} I_{\mathrm{p}}(1-R)\right)+b_{i} c_{i} \theta_{i}(1-R)\right]\right]^{2}-4 \sum_{i=1}^{n} b_{i} \times\left[\frac{1}{T^{3}}\left(S+\sum_{i=1}^{n} s_{i}\right)+\sum_{i=1}^{n} \frac{\left(a_{i}-b_{i} p_{i}\right) t_{d i}}{T^{3}\left(\lambda_{i}+\theta_{i}\right)}\left(h_{i}+c_{i} I_{\mathrm{p}}(1-R)\right)\right]
\end{aligned}
$$

Theorem 1 If $1-R \in[0,1]$, there are optimal values of $p$ and $T$ for maximum value of the total profit $T P^{1}$. 
Theorem 2 if $n+\sum_{i=1}^{n} \theta_{i} \mathrm{~T}+\frac{I_{p} t_{d i}}{T\left(\lambda_{i}+\theta_{i}\right)}>\sum_{i=1}^{n} 2\left(I_{p}-\theta_{i}\right) t_{d i}$, retailer's profit is proportional to the price discount rate; if $n+\sum_{i=1}^{n} \theta_{i} \mathrm{~T}+\frac{I_{p} t_{d i}}{T\left(\lambda_{i}+\theta_{i}\right)} \leq \sum_{i=1}^{n} 2\left(I_{p}-\theta_{i}\right) t_{d i}$, retailer's profit is inversely proportional to the price discount rate.

Let Eq.(8) and Eq.(9) to be zero, we obtain the optimal value of $p_{i}^{1^{*}}$ and $T$ in price discount:

$p_{i}^{1 *}=\frac{1}{2 b_{i}}\left[a_{i}+b_{i} c_{i}(1-R)\left(1-\theta_{i} t_{d i}\right)+\frac{b_{i} t_{d i}\left(1-2\left(\lambda_{i}+\theta_{i}\right) T\right)}{T\left(\lambda_{i}+\theta_{i}\right)}\left(h_{i}+c_{i} I_{\mathrm{p}}(1-R)\right)+b_{i} c_{i} \theta_{i}(1-R)\left(T-t_{d i}\right)\right]$

$T^{1^{*}}=\sqrt{\frac{S+\sum_{i=1}^{n} s_{i}+\sum_{i=1}^{n} t_{d i}\left(a_{i}-b_{i} p_{i}\right)\left(h_{i}+c_{i} I_{\mathrm{p}}(1-R)\right) /\left(\lambda_{i}+\theta_{i}\right)}{\sum_{i=1}^{n} c_{i} \theta_{i}(1-R)\left(a_{i}-b_{i} p_{i}\right)}}$

According to Theorem1, we get:

$$
R^{*}= \begin{cases}\bar{R} & n+\sum_{i=1}^{n} \theta_{i} T+\frac{I_{p} t_{d i}}{T\left(\lambda_{i}+\theta_{i}\right)}>\sum_{i=1}^{n} 2\left(I_{p}-\theta_{i}\right) t_{d i} \\ 0 & n+\sum_{i=1}^{n} \theta_{i} T+\frac{I_{p} t_{d i}}{T\left(\lambda_{i}+\theta_{i}\right)}<\sum_{i=1}^{n} 2\left(I_{p}-\theta_{i}\right) t_{d i}\end{cases}
$$

3.2 Results under trade credit

First of all, we should to determine the $\left(p_{i}, T\right)$ of item i is not only exist but the only for any $M$. For any $p_{i}$ and $T$, the $M$ is not only exist but the only.

Scenario 1 if $M<T$, there is an optimal solution of the profits.

Taking the partial derivative of $T P^{2}$ with respective to $p$ and $T$, we have:

$$
\begin{gathered}
\frac{\partial T P^{2}}{\partial p}=\sum_{i=1}^{n}\left\{\left(a_{i}-2 b_{i} p_{i}\right)-\frac{b_{i} p_{i} I_{e} M}{T}+b_{i} c_{i}\left(1-\theta_{i} t_{d i}\right)(1-r M)+\frac{b_{i} h_{i} t_{d i}}{\lambda_{i}+\theta_{i}}\left(\frac{1}{T}-2\left(\lambda_{i}+\theta_{i}\right)\right)+\frac{b_{i} c_{i} \theta_{i}}{\lambda_{i}+\theta_{i}}\left[\frac{2 t_{d i}}{T}-\left(\lambda_{i}+\theta_{i}\right) T+\left(\lambda_{i}+\theta_{i}\right) t_{d i}\right]+\right. \\
Y_{i} b_{i} c_{i} I_{p}\left[\left(t_{d i}-M\right)\left(1-\theta_{i} t_{d i}+\frac{\theta_{i} t_{d i}^{2}}{T}\right)+1\right]+\left(1-Y_{i}\right) \frac{b_{i} c_{i} I_{p}}{\left(\lambda_{i}+\theta_{i}\right)\left(\theta_{i}+\mathrm{r}\right)}\left[\left(\lambda_{i}+\theta_{i}\right) T-\frac{\left(1-\theta_{i}-r\right) M}{T}+1-\theta_{i}-r-M\left(\lambda_{i}+\theta_{i}\right)\right] \\
\frac{\partial T P^{2}}{\partial T}=\sum_{i=1}^{n}\left[\frac{-p_{i} I_{e}\left(a_{i}-b_{i} p_{i}\right) M}{T^{2}}+\frac{\left(h_{i}+2 c_{i} \theta_{i}\right)\left(a_{i}-b_{i} p_{i}\right) t_{d i}}{T^{2}\left(\lambda_{i}+\theta_{i}\right)}+Y_{i} c_{i} \theta_{i}\left(a_{i}-b_{i} p_{i}\right)+\frac{Y_{i} c_{i} \theta_{i} I_{p}\left(a_{i}-b_{i} p_{i}\right) t_{d i}^{2}}{T^{2}}\left(t_{d i}-M\right)-\right. \\
\left.\left(1-Y_{i}\right) \frac{c_{i} I_{p}\left(a_{i}-b_{i} p_{i}\right)}{\theta_{i}+r}-\left(1-Y_{i}\right) \frac{c_{i} I_{p}\left(a_{i}-b_{i} p_{i}\right)\left(1-\theta_{i}-r\right) M}{T^{2}\left(\lambda_{i}+\theta_{i}\right)\left(\theta_{i}+r\right)}\right]+\frac{1}{T^{2}}\left(S+\sum_{i=1}^{n} s_{i}\right)
\end{gathered}
$$

Taking the second partial derivative of $T P^{2}$ with respective to $p$ and $T$, and we have the Hessian matrix:

$$
\begin{aligned}
\Delta\left(H^{2}\right)= & \left\{\sum_{i=1}^{n}\left[\frac{b_{i} p_{i} I_{e} M}{T^{2}}-\frac{b_{i} t_{d i}\left(h_{i}+2 c_{i} \theta_{i}\right)}{T^{2}\left(\lambda_{i}+\theta_{i}\right)}-Y_{i} b_{i} c_{i} \theta_{i}-\frac{Y_{i} b_{i} c_{i} \theta_{i} I_{p} t_{d i}^{2}}{T^{2}}\left(t_{d i}-M\right)+\left(1-Y_{i}\right) \frac{b_{i} c_{i} I_{p}}{\theta_{i}+r}+\left(1-Y_{i}\right) \frac{b_{i} c_{i} I_{p}\left(1-\theta_{i}-\mathrm{r}\right) M}{T^{2}\left(\lambda_{i}+\theta_{i}\right)\left(\theta_{i}+r\right)}\right]\right\}^{2} \\
& -4 \sum_{i=1}^{n} b_{i} \times\left\{\sum _ { i = 1 } ^ { n } \left[\frac{-p_{i} I_{\mathrm{e}}\left(a_{i}-b_{i} p_{i}\right) M}{T^{3}}+\frac{\left(h_{i}+2 \mathrm{c}_{i} \theta_{i}\right)\left(a_{i}-b_{i} p_{i}\right) t_{d i}}{T^{3}}+\frac{Y_{i} \theta_{i} I_{\mathrm{p}}\left(a_{i}-b_{i} p_{i}\right) t_{d i}^{2}}{T^{3}}\left(t_{d i}-M\right)-\right.\right. \\
& \left.\left.\left(1-Y_{i}\right) \frac{c_{i} I_{\mathrm{p}}\left(a_{i}-b_{i} p_{i}\right)\left(1-\theta_{i}-r\right) M}{T^{3}\left(\lambda_{i}+\theta_{i}\right)\left(\theta_{i}+r\right)}\right]+\frac{1}{T^{3}}\left(S+\sum_{i=1}^{n} s_{i}\right)\right\}
\end{aligned}
$$

Theorem 3 if $M<T$, retailer always get the optimum values for any $M$.

Scenario 2 if $M>T$, there is an optimal solution of the profits.

Taking the partial derivative of $T P^{3}$ with respective to $p$ and $T$, we have:

$$
\begin{gathered}
\frac{\partial T P^{3}}{\partial p}=\sum_{i=1}^{n} a_{i}-2 b_{i} p_{i}-b_{i} p_{\mathrm{i}} I_{e}(T-M+1)+b_{i} c_{i}(1-r M)\left(1-\theta_{i} t_{d i}\right)+\frac{b_{i} h_{i} t_{d i}}{\lambda_{i}+\theta_{i}}\left(\frac{1}{T}-2\left(\lambda_{i}+\theta_{\mathrm{i}}\right)\right)+\frac{b_{i} c_{i} \theta_{i}}{\lambda_{i}+\theta_{i}}\left[\frac{2 t_{d i}}{T}-\left(\lambda_{i}+\theta_{i}\right) \mathrm{T}+\left(\lambda_{i}+\theta_{i}\right) t_{d i}-2\right] \\
\frac{\partial T P^{3}}{\partial T}=\sum_{i=1}^{n}\left[p_{i} I_{e}\left(a_{i}-b_{i} p_{i}\right)+\frac{\left(h_{i}+2 c_{i} \theta_{i}\right)\left(a_{i}-b_{i} p_{i}\right) t_{d i}}{T^{2}\left(\lambda_{i}+\theta_{i}\right)}+c_{i} \theta_{i}\left(a_{i}-b_{i} p_{i}\right)\right]+\frac{1}{T^{2}}\left(S+\sum_{i=1}^{n} s_{i}\right)
\end{gathered}
$$

Taking the second partial derivative of $T P^{3}$ with respective to $p$ and $T$, and we have the Hessian matrix: 


$$
\begin{aligned}
\Delta\left(H^{3}\right) & =\left[\sum_{i=1}^{n}\left(b_{i} p_{i} I_{e}-\frac{b_{i} t_{d i}\left(h_{i}+2 c_{i} \theta_{i}\right)}{T^{2}\left(\lambda_{i}+\theta_{i}\right)}-b_{i} c_{i} \theta_{i}\right)\right]^{2}-2 \sum_{i=1}^{n} b_{i} \times\left[\sum_{i=1}^{n}\left[\frac{\left(2 h_{i}+4 c_{i} \theta_{i}\right)\left(a_{i}-b_{i} p_{i}\right) t_{d i}}{T^{3}\left(\lambda_{i}+\theta_{i}\right)}\right]+\frac{2}{T^{3}}\left(S+\sum_{i=1}^{n} s_{i}\right)\right] \\
& \left.=\sum_{i=1}^{n} b_{i} \times\left\{\mathrm{b}_{i} \sum_{i=1}^{n}\left(p_{i} I_{e-} c_{i} \theta_{i}-\frac{b_{i} t_{d i}\left(h_{i}+2 c_{i} \theta_{i}\right)}{T^{2}\left(\lambda_{i}+\theta_{i}\right)}\right)\right]^{2}-4 \sum_{i=1}^{n}\left[\frac{\left(h_{i}+2 c_{i} \theta_{i}\right)\left(a_{i}-b_{i} p_{i}\right) t_{d i}}{T^{3}\left(\lambda_{i}+\theta_{i}\right)}\right]-\frac{4}{T^{3}}\left(S+\sum_{i=1}^{n} s_{i}\right)\right\}
\end{aligned}
$$

Theorem 4 if $T^{3} b_{i} \sum_{i=1}^{n}\left(p_{i} I_{e}-c_{i} \theta_{i}\right)^{2}<8\left(S+\sum_{i=1}^{n} s_{i}\right)$, or any $M$, the retailer can obtain the optimal value.

When $M<T$, let Eq.(11) and Eq.(12) equal to zero, we have:

$$
\begin{aligned}
& p_{i}^{2^{*}}=\frac{1}{2 b_{i}}\left\{a_{i}-\frac{b_{i} p_{i} L_{e} M}{T}+b_{i} c_{i}\left(1-\theta_{i} t_{d i}\right)(1-r M)+\frac{b_{i} h_{i} t_{d i}}{\lambda_{i}+\theta_{i}}\left(\frac{1}{T}-2\left(\lambda_{i}+\theta_{i}\right)\right)+\frac{b_{i} c_{i} \theta_{i}}{\lambda_{i}+\theta_{i}}\left[\frac{2 t_{d i}}{T}-\left(\lambda_{i}+\theta_{i}\right) T+\left(\lambda_{i}+\theta_{i}\right) t_{d i}\right]+\right. \\
& \left.Y_{i} b_{i} c_{i} I_{p}\left[\left(t_{d i}-M\right)\left(1-\theta_{i} t_{d i}+\frac{\theta_{i} t_{d i}^{2}}{T}\right)+1\right]+\left(1-Y_{i}\right) \frac{b_{i} c_{i} I_{p}}{\left(\lambda_{i}+\theta_{i}\right)\left(\theta_{i}+\mathrm{r}\right)}\left[\left(\lambda_{i}+\theta_{i}\right) T-\frac{\left(1-\theta_{i}-r\right) M}{T}+1-\theta_{i}-r-M\left(\lambda_{i}+\theta_{i}\right)\right]\right\} \\
& T^{2^{*}}=\sqrt{\frac{S+\sum_{i=1}^{n} s_{i}+\sum_{i=1}^{n}\left(a_{i}-b_{i} p_{i}\right) \times\left[\frac{\left(h_{i}+2 c_{i} \theta_{i}\right) t_{d i}}{\lambda_{i}+\theta_{i}}-p_{i} I_{e} M+Y_{i} c_{i} \theta_{i} I_{p} t_{d i}^{2}\left(t_{d i}-M\right)-\left(1-Y_{i}\right) \frac{c_{i} I_{p}\left(1-\theta_{i}-\mathrm{r}\right) M}{\left(\lambda_{i}+\theta_{i}\right)\left(\theta_{i}+r\right)}\right]}{\sum_{i=1}^{n} c_{i} I_{p}\left(1-Y_{i}\right)\left(a_{i}-b_{i} p_{i}\right) /\left(\theta_{i}+r\right)-Y_{i} c_{i} \theta_{i}\left(a_{i}-b_{i} p_{i}\right)}}
\end{aligned}
$$

According to $d T P^{2} / d M$ and $d T P^{3} / d M$, we have:

$$
M^{2^{*}}=\left\{\begin{array}{ll}
T & \sum_{i=1}^{n} r c_{i}\left(1-\theta_{i} t_{d i}\right)<\sum_{i=1}^{n} p_{i} I_{e} \\
\bar{M} & \sum_{i=1}^{n} r c_{i}\left(1-\theta_{i} t_{d i}\right)>\sum_{i=1}^{n} p_{i} I_{e}
\end{array} \text { and } M^{3^{*}}= \begin{cases}T & \sum_{i=1}^{n} r c_{i}\left(1-\theta_{i} t_{d i}\right)<\sum_{i=1}^{n} p_{i} I_{e} \\
\bar{M} & \sum_{i=1}^{n} r c_{i}\left(1-\theta_{i} t_{d i}\right)>\sum_{i=1}^{n} p_{i} I_{e}\end{cases}\right.
$$

And when $M \geq T$, let Eq.(14) and Eq.(15) equal to zero, we have:

$$
\begin{aligned}
& p_{i}^{3^{*}}=\frac{1}{2 b_{i}}\left\{a_{i}-b_{i} p_{\mathrm{i}} I_{e}(T-M+1)+b_{i} c_{i}(1-r M)\left(1-\theta_{i} t_{d i}\right)+\frac{b_{i} h_{i} t_{d i}}{\lambda_{i}+\theta_{i}}\left(\frac{1}{T}-2\left(\lambda_{i}+\theta_{\mathrm{i}}\right)\right)+\frac{b_{i} c_{i} \theta_{i}}{\lambda_{i}+\theta_{i}}\left[\frac{2 t_{d i}}{T}-\left(\lambda_{i}+\theta_{i}\right) \mathrm{T}+\left(\lambda_{i}+\theta_{i}\right) t_{d i}-2\right\}\right. \\
& T^{3^{*}}=\sqrt{\frac{-S-\sum_{i=1}^{n} s_{i}-\sum_{i=1}^{n}\left(h_{i}+2 c_{i} \theta_{i}\right)\left(a_{i}-b_{i} p_{i}\right) t_{d i} /\left(\lambda_{i}+\theta_{i}\right)}{\sum_{i=1}^{n}\left(p_{i} I_{e}+c_{i} \theta_{i}\right)\left(a_{i}-b_{i} p_{i}\right)}}
\end{aligned}
$$

Above all, the retailer's optimal value can be summarized as:

$$
T P M^{*}=\operatorname{Max}\left\{\begin{array}{lc}
T P^{2^{*}}\left(p_{i}^{*}, T^{2 *}, \mathrm{M}^{2^{*}}\right) & M<t_{d i}<T \\
\operatorname{TP}^{3^{*}}\left(p_{i}^{*}, T^{3^{*}}, \mathrm{M}^{3^{*}}\right) & t_{d i}<T<M
\end{array}\right.
$$

If $\mathrm{TPM}^{*}<T P^{*}$, retailer should be ordering in the form of price discount;

If $\mathrm{TPM}^{*}>T P^{i^{*}}$, retailer should be ordering in the form of trade credit;

If $\mathrm{TPM}^{*}=T P^{\mathbf{l}^{*}}$, if the type of retailer is limited funds, small and medium-sized enterprises, he should take the way of the trade credit to ensure that the flounce of internal cash flow.

$$
\text { 4. Numerical examples }
$$

We solve the model using the Matlab2010. We chose two items, and the parameters as following:

Item 1: $S=300, s_{1}=0, c=20, h=10, R=\sum_{i=1}^{n} e^{-Q} \rightarrow 0.58, D(p, \mathrm{t})=(600-2 p) e^{-0.98 t}, \theta=0.08, r=0.10, I_{e}=0.12, I_{p}=0.15$, $t_{d}=1 / 12$.

Item 2: $s_{2}=10, c=30, h=12, R=\sum_{i=1}^{n} e^{-Q} \rightarrow 0.58, D(p, \mathrm{t})=(500-p) e^{-0.98 t}, \theta=0.05, r=0.10, I_{e}=0.12, I_{p}=0.15, t_{d}=1 / 10$.

5.1 Numerical example analysing for price discount

According to parameters value, we can acquire the result of inventory ordering policies under price discount, as describe in table1. 
Table1 Computational result under price discount

\begin{tabular}{ccccccc}
\hline$R$ & $p_{1}{ }^{*}$ & $p_{2}{ }^{*}$ & $T^{*}$ & $Q_{1}{ }^{*}$ & $Q{ }^{*}$ & $T P^{1^{*}}$ \\
\hline 0 & $\mathbf{1 5 8 . 5 7 5 9}$ & $\mathbf{2 6 2 . 5 7 3 6}$ & $\mathbf{0 . 7 4 7 4}$ & 210.14 & 176.677 & 93839.3 \\
0.1 & 157.6166 & 261.1643 & 0.7724 & 218.656 & 183.683 & 95147 \\
0.2 & 156.6585 & 259.7567 & 0.8027 & 228.732 & 191.988 & 96467.3 \\
0.3 & 155.7017 & 258.3511 & 0.8399 & 240.932 & 202.063 & 97801.1 \\
0.4 & 154.7466 & 256.9482 & 0.8871 & 256.143 & 214.647 & 99149.7 \\
0.45 & 154.2699 & 256.248 & 0.9158 & 265.308 & 222.239 & 99830.1 \\
0.5 & 153.7937 & 255.5488 & 0.9492 & 275.861 & 230.988 & 100515 \\
0.55 & 153.3184 & 254.8507 & 0.9884 & 288.188 & 241.215 & 101204.8 \\
0.6 & 152.8438 & 254.154 & 1.0353 & $\mathbf{3 0 2 . 8 4 2}$ & $\mathbf{2 5 3 . 3 8 1}$ & $\mathbf{1 0 1 8 9 9 . 8}$ \\
\hline
\end{tabular}

According to table 1, the price of the two non-instantaneous deterioration items decrease with the price discount rate; the ordering quantity of the two non-instantaneous deterioration items increase with the price discount rate. Under the coordination order cycle, due to different deterioration rate and deterioration time will lead to different retailer's strategy of different items order quantity and price. It means if the deterioration time is shorter, the retailer will order more item s and the price will be lower. The profit and order period are increasing with the discount rate.

5.2 Numerical example analysing for trade credit

Scenario 1: when the trade credit in the interval of $[0, T]$, the result can be seen in Fig.4. According to theorem 4, if $M<\mathrm{T}$, the optimal ordering period is $T^{2 *}=0.373$. According to Fig.4, the profit is increasing with trade credit, and the optimal profit is $T P^{2^{*}}=107066.7$, when $M^{2^{*}}=T$. So the two kinds items' inventory ordering parameters are $p_{1}^{*}=155.15$, $p_{2}{ }^{*}=260.39, Q_{1}{ }^{*}=107.48, Q_{2}{ }^{*}=89.04$.

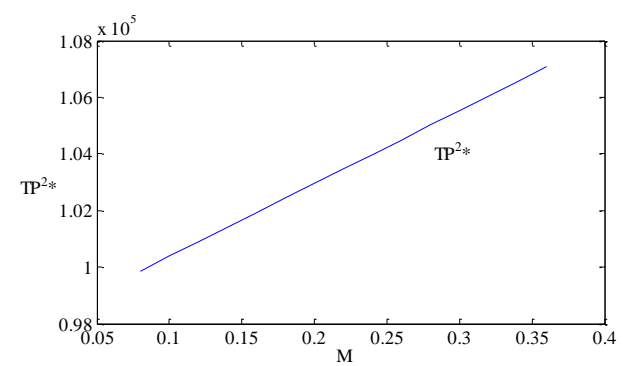

Fig. 4 when $M<\mathrm{T}$, the trend of profit

Scenario 2: when the trade credit in the interval of $[\mathrm{T},+\infty]$, the result can be seen in table 2 and Fig.5.

Table2 when $M>\mathrm{T}$, the result of retailer under trade credit

\begin{tabular}{ccccccc}
\hline$M$ & $p_{1}{ }^{*}$ & $p_{2}{ }^{*}$ & $T^{*}$ & $Q_{1}{ }^{*}$ & $Q_{2}{ }^{*}$ & $T P^{3 *}$ \\
\hline 0.25 & 149.1053 & 246.8186 & 0.2495 & 74.972 & 62.9878 & $\mathbf{1 0 7 2 4 4 . 2}$ \\
0.252 & 149.1203 & 246.8438 & 0.2495 & 74.9578 & 62.9759 & 107222.1 \\
0.254 & 149.1353 & 246.869 & 0.2495 & 74.9436 & 62.964 & 107200.1 \\
0.256 & 149.1504 & 246.8942 & 0.2495 & 74.9295 & 62.9521 & 107178.1 \\
0.258 & 149.1654 & 246.9194 & 0.2494 & 74.9153 & 62.9401 & 107156 \\
0.26 & 149.1804 & 246.9446 & 0.2494 & 74.9011 & 62.9282 & 107133.9 \\
0.261 & 149.1879 & 246.9572 & 0.2494 & 74.894 & 62.9223 & 107122.9 \\
0.262 & 149.1954 & 246.9698 & 0.2494 & 74.8869 & 62.9163 & 107112 \\
\hline
\end{tabular}




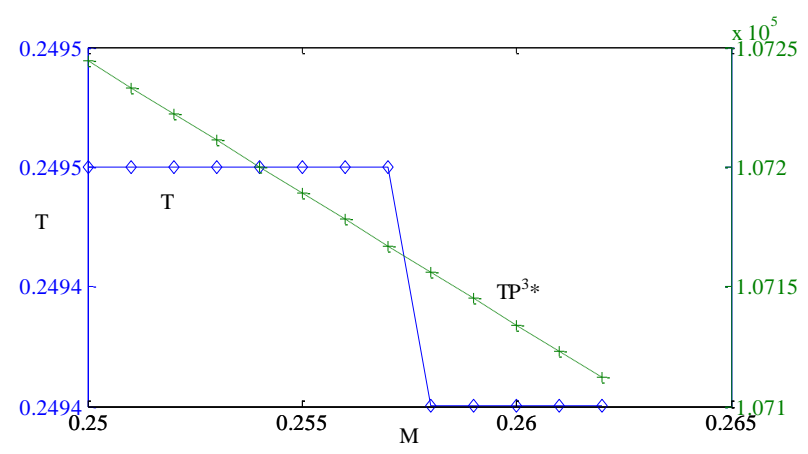

Fig. 5 when $M>\mathrm{T}$, the trend of profit and ordering period

According to table 2, if $M>\mathrm{T}$, the ordering quantity and price is stable of the two kinds non-instantaneous deterioration items. When $M \leq 0.256$, we get $T^{*}=0.295$; when $M \geq 0.258$, we get $T^{*}=0.294$. From Fig. 5 , the profit is decreasing with trade credit. So retailer will obtain the optimal value at the point of $M=\mathrm{T}$. And the profit value is $T P^{3^{*}}=107244.2$.

Table 3 cooperation of different circumstance

\begin{tabular}{|c|c|c|c|c|c|c|c|c|c|c|}
\hline & \multirow{2}{*}{$R^{*}$} & \multirow{2}{*}{$M^{*}$} & \multirow{2}{*}{$p_{1}{ }^{*}$} & \multirow{2}{*}{$p_{2}{ }^{*}$} & \multirow{2}{*}{$Q_{1}{ }^{*}$} & \multirow{2}{*}{$Q_{2}{ }^{*}$} & \multirow{2}{*}{$T^{*}$} & \multirow{2}{*}{$T P^{1^{*}}$} & \multicolumn{2}{|c|}{$T P M^{*}$} \\
\hline & & & & & & & & & $T P^{2^{*}}$ & $T P^{3^{*}}$ \\
\hline Price discount & 0.6 & - & 152.84 & 254.15 & 302.84 & 253.3 & 1.035 & 101899.8 & - & - \\
\hline Trade credit & - & 0.25 & 149.11 & 246.82 & 74.97 & 62.99 & 0.25 & - & 107066.7 & 107244.2 \\
\hline
\end{tabular}

From table 3 , it is easy to find that $T P^{1^{*}}<T P M$. So when $t_{d}<T$, the profit of retailer under trade credit is better than that of price discount. And in the trade credit, the profit under $M<\mathrm{T}$ is better than under $M>\mathrm{T}$. This is because of the former has bigger ordering quantity. Also, when $0.25<T<0.36$, it is better for retailer to order less. Fig. 6 describes the change of profit, and shows that the optimal value are $M^{*}=0.25, p_{1}^{*}=149.11, p_{2}^{*}=246.82, Q_{1}^{*}=74.97, Q_{2}^{*}=62.99, T^{*}=0.25$, $T P=T P M=107244.2$.

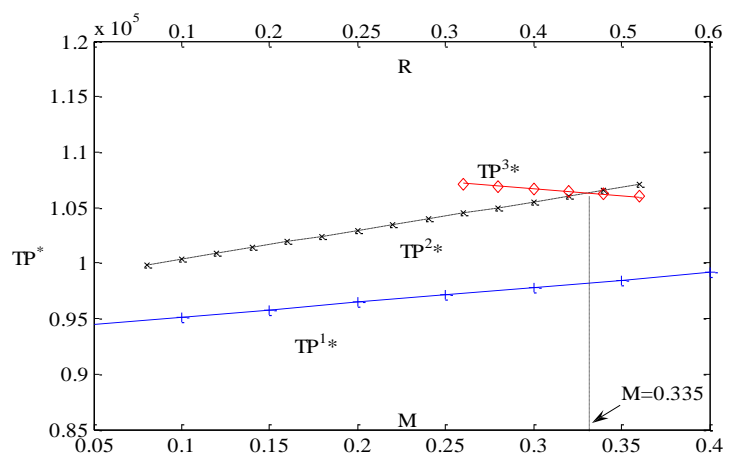

Fig.6 the trend of profit under different circumstance

Conclusions

In this paper, we build up an inventory ordering model for non-instantaneously deteriorating items based on trade credit and price discount. First, established inventory ordering model under different situations by maximize retailer total revenue; then through the numerical calculation to prove the existence of the optimal solution, and obtain the optimal solution; lastly, through numerical calculate out: the retailer's profit increases with the price discount rate or the trade credit $(M<\mathrm{T})$; the retailer's profit decreases with the trade credit $(M>\mathrm{T})$; and the retailer's profit under trade credit is better than price discount, when multiple non-instantaneously deteriorating items are ordered coordination. The retailer will be obtain his optimal profit, when $M=0.25$. What's more, this work can be extended in several ways: for instance, discussing the circumstances of $t_{d}>T$, uncertain demands could be considered by allowing the demand function to be driven by probability, since uncertain issues are very important on policy or decision making (Sun et al., 2018). In addition, a constant or random ordering lead time could be considered. 


\section{ACKNOWLEDGEMENTS}

This work was supported by the National Social Science Foundation of China [17CGL019] and the Humanities and Social Sciences Program of Shandong Universities [J17RB094].

\section{REFERENCES}

[1] Bose, S., Goswami, A., Chaudhuri, K. S. 1995. An EOQ model for deteriorating items with linear time-dependent demand rate and shortages under inflation and time discounting. Journal of the Operational Research Society, 46(6), $771-782$.

[2] Chang, C. T., Teng, J. T., Goyal, S. K. 2010. Optimal replenishment policies for non-instantaneous deteriorating items with stockdependent demand. International Journal of Production Economics, 123(1), 62-68.

[3] Dye, C. Y. 2013. The effect of preservation technology investment on a non-instantaneous deteriorating inventory model. Omega, 41(5), 872-880.

[4] Li, M. F., Wang, D. P. 2011. Inventory Model for Non-Instantaneous Deteriorating Items under Permissible Payment Delay. Industrial Engineering Journal, 14(6), 38-43.

[5] Maihami, R., Abadi, I. N. K. 2012. Joint control of inventory and its pricing for non-instantaneously deteriorating items under permissible delay in payments and partial backlogging. Mathematical and Computer Modelling, 55(5-6), 1722-1733.

[6] Maihami, R., Kamalabadi, I. N. 2012. Joint pricing and inventory control for non-instantaneous deteriorating items with partial backlogging and time and price dependent demand. International Journal of Production Economics, 136(1), 116-122.

[7] Mo, J. T., Chen, G. M., Fan, T., Mao, H. 2011. The optimal replenishment strategy for perishable multi-products with stock-dependent and capacity constraint. Systems engineering, 29, 98-101.

[8] Moon, I., Giri, B. C., Ko, B. 2005. Economic order quantity models for ameliorating/deteriorating items under inflation and time discounting. European Journal of Operational Research, 162(3), 773-785.

[9] Musa, A., Sani, B. 2012. Inventory ordering policies of delayed deteriorating items under permissible delay in payments. International Journal of Production Economics, 136(1), 75-83.

[10] Shah, N. H., Soni, H. N., Patel, K. A. 2013. Optimizing inventory and marketing policy for non-instantaneous deteriorating items with generalized type deterioration and holding cost rates. Omega, 41(2), 421-430.

[11] Sun, Y., Hrušovský, M., Zhang, C., Lang, M. 2018. A Time-Dependent Fuzzy Programming Approach for the Green Multimodal Routing Problem with Rail Service Capacity Uncertainty and Road Traffic Congestion[J]. Complexity, 2018:1-22.

[12] Tsao, Y. C., Sheen, G. J. 2012. A multi-item supply chain with credit periods and weight freight cost discounts. International Journal of Production Economics, 135(1), 106-115.

[13] Tsao, Y. C. 2010. Managing multi-echelon multi-item channels with trade allowances under credit period. International Journal of Production Economics, 127(2), 226-237.

[14] Wang Chuanxu, Jiang Liangkui 2007. Price and Ordering Cycle Decision in a Supply Chain with Multiple Deteriorating Goods. Journal of Operational Research, 11(5):86-92.

[15] Wen Xiaowei, Da Liqing 2006. Study on Ordering Policy of Multi-product in Deteriorating Items Supply Chain. Systems engineering theory and Practice, 43(2):43-48. (in Chinese)

[16] Wu, K. S., Ouyang, L. Y., Yang, C. T. 2006. An optimal replenishment policy for non-instantaneous deteriorating items with stockdependent demand and partial backlogging. International Journal of Production Economics, 101(2), 369 -384.

[17] Yang, H. L., Chang, C. T. 2013. A two-warehouse partial backlogging inventory model for deteriorating items with permissible delay in payment under inflation. Applied Mathematical Modelling, 37(5), 2717-2726.

[18] Zhang, J. R., Yu, Y. L., Zhang, L., Qu, C. Z., Su, Z. H. 2012. Joint replenishment policy of multi-item inventory. Computer Integrated Manufacturing Systems, 18(6), 1246-1256. 